\title{
AVALIAÇÃO DE TRATAMENTOS QUÍMICOS E MECÂNICOS NO CONTROLE DE PLANTAS DANINHAS NA CULTURA DO ALGODÃO ${ }^{1}$
}

\author{
LUIZ L. FOLONI ${ }^{2}$, JOÃO D. RODRIGUES ${ }^{3}$ e ELIZABETH O. ONO ${ }^{4}$
}

\section{RESUMO}

O presente trabalho teve como objetivo avaliar a eficiência de diferentes combinações de tratamentos herbicidas integrado, aplicados em PPI, pré, pós, cultivo mecânico e jato dirigido em cultura de algodão (Gossypium hirsutum L.). O experimento foi conduzido em campo, sendo a área experimental instalada na cultura de algodão cultivar IAC-20. Foram testados tratamentos com os seguintes produtos: trifluralina na dose de 1,068 kg i.a./ha; alachlor na dose de $1,584 \mathrm{~kg}$ i.a./ha; M.S.M.A. nas doses de 1,44 e $1,68 \mathrm{~kg}$ i.a./ha; nicosulfuron nas doses de 0,0201 ; 0,030 e $0,040 \mathrm{~kg}$ i.a./ha; diuron nas doses de $1,0 \mathrm{e}$ $1,5 \mathrm{~kg}$ i.a./ha; lactofen na dose de $0,18 \mathrm{~kg}$ i.a./ha; cyanazine nas doses de 1,0 e $1,5 \mathrm{~kg}$ i.a./ha e diuron nas doses de 1,0 e 1,5 kg i.a./ha. Os tratamentos herbicidas foram efetuados em pré-plantio incorporado (PPI), pré-emergência (PRE), pósemergência (POS), cultivo mecânico (CM) e jato dirigido (JD). Os resultados obtidos levaram a concluir que o sistema de trifluralina (PPI), alachlor (PRE), CM, M.S.M.A. + lactofen apresentaram a maior produtividade. Os sistemas herbicidas compostos por aplicação de trifluralina (PPI), alachlor (PRE), M.S.M.A. (POS), CM ao redor dos 50 a 60 D.A.P. (dias após o plantio) e a aplicação de jato dirigido de diuron, lactofen + cyanazine, lactofen + diuron, M.S.M.A. + cyanazine, M.S.M.A. + lactofen e M.S.M.A. + diuron, propiciaram de forma geral controle das principais plantas daninhas.

Palavras chave: Gossypium hirsutum, fitotoxicidade, controle, herbicidas.

\section{ABSTRACT}

\section{Mechanical and chemical treatments in the weed controls in the cotton crop}

The objective of this work was to evaluate the efficacy of different combinations of integrated herbicide treatments, applied at PPI, pre, post, mechanical cultivation and direct spray on cotton crop (Gossypium hirsutum). The trial was conducted in the field, being cotton crop cultivar IAC-20 the experiment area. The treatments were tested with the following products: trifluralina at the dose of $1.068 \mathrm{~kg}$ a.i./ha; alachlor at the dose of $1.584 \mathrm{~kg}$ a.i/ha; M.S.M.A. at the doses of 1.44 and $1.68 \mathrm{~kg}$ a.i./ha; nicosulfuron at the doses of $0.0201 ; 0.030$ and $0.040 \mathrm{~kg}$ a.i./ha; diuron at the doses of 1.0 and $1.5 \mathrm{~kg}$ a.i./ha; lactofen at the dose of $0,18 \mathrm{~kg}$ a.i./ha; cyanazine at the doses of 1.0 and $1.5 \mathrm{~kg}$ a.i./ha and diuron at the doses of 1.0 and $1.5 \mathrm{~kg}$ a.i./ha. The herbicide treatments were conducted at incorpored preplanting (IPP), preemergence (PRE), postemergence (POS); mechanical cultivation (MC) and directed spray (DS). The results lead to the conclusion that the system trifluralina (IPP), alachlor (PRE), MC, M.S.M.A. + lactofen presented the highest yielding.

\footnotetext{
${ }^{1}$ Recebido para publicação em 30/04/98 na forma revisada em 03/09/98.

${ }^{2}$ Professor Colaborador do Curso de Pós-Graduação do Departamento de Água e Solo, da Faculdade de Engenharia Agrícola/UNICAMP.

${ }^{3}$ Professor Titular, Departamento de Botânica, IB/UNESP. CEP 18618-000, Botucatu/SP.

${ }^{4}$ Professora Assistente Dr ${ }^{\mathrm{a}}$, Departamento de Botânica, IB/UNESP. CEP 18618-000, Botucatu/SP.
} 
The herbicide systems with the application of trifluralina (IPP), alachlor (PRE), M.S.M.A. (POS), MC approximately 50 to 60 D.A.P. and the application through direct spray of diuron, lactofen

\section{INTRODUÇ̃̃O}

O algodoeiro é uma das culturas econômicas mais suscetíveis à concorrência de plantas daninhas, que a prejudicam desde o plantio até a colheita. Em casos extremos, o prejuízo pode chegar a $90 \%$. Na fase inicial da cultura reduzem bastante o crescimento e vigor das plantas, além de hospedar pragas e doenças. No final do ciclo causam perdas, devido à redução na qualidade da fibra, dificultando a colheita manual ou mecânica e ocasionando baixo rendimento de trabalho, assim como reduzem a eficiência das máquinas beneficiadoras, devido a fibras imaturas, aumento de refugo ("carimã"), tornando difícil o descaroçamento. Aumentam a umidade das sementes, atrasando a colheita e reduzindo a qualidade do línter. Muitas plantas daninhas contém em suas folhas alto teor de sílica, o que pode prejudicar a fibra, funcionando como "fibras duras", que causam sérios prejuízos na fiação (Laca-Buendia, 1992).

Os métodos de controle para a eliminação total ou parcial das plantas daninhas podem ser manuais, mecânicos (animal ou tratorizado) e químicos. O uso de enxadas tem-se mostrado inadequado, tanto pela baixa disponibilidade de pessoal como pelo baixo rendimento e eficiência no controle das plantas daninhas no período crítico de concorrência, o qual normalmente coincide com períodos de alta pluviosidade.

O cultivo mecânico, apesar de mais econômico que o manual, mostra-se também pouco eficaz, devido ao não controle das plantas daninhas próximos a linha de plantio, podendo causar danos tanto ao caule como no sistema radicular do algodoeiro, reduzindo assim, a capacidade produtiva.

Desde os trabalhos pioneiros de Leiderman et al. (1965) que estudaram a eficiência
+ cyanazine, lactofen + diuron, M.S.M.A. + cyanazine, M.S.M.A. + lactofen and M.S.M.A. + diuron, in general, promoted the control of weeds.

Key words: Gossypium hirsutum, herbicides, control, phytotoxicity.

da trifluralina e do diuron e de Alves \& Forster (1968) que estudaram os métodos de aplicação, centenas de outras pesquisas foram desenvolvidas com as mais diferentes finalidades, como avaliar a eficiência de misturas, o controle de determinadas espécies, fitotoxicidade, dosagens, tipo de aplicação, etc.

O cultivo químico em algodão pode ser realizado em pré-plantio incorporado, préemergência e pós-emergência. Rhodes \& Shelby (1993) no trabalho de controle de plantas daninhas em algodão faz referência, além dos três tipos de aplicação citados anteriormente, de um quarto tipo. Chamam este de aplicação "early overtop" (salvage use) em aplicação em área total na pósemergência precoce, ou ainda, "uso de salvação". Os herbicidas utilizados neste tipo de controle são o fluometuron, D.S.M.A. e M.S.M.A., sendo especificamente o M.S.M.A. recomendado em aplicação em área total, com o algodão entre $7 \mathrm{e}$ $15 \mathrm{~cm}$ de altura para o controle emergencial (uso de salvação) de folhas largas e gramíneas.

A combinação apropriada de herbicidas em pré-plantio incorporado (PPI) ou foliar, de contato, pré-emergentes, pós-emergentes e outros herbicidas com outras técnicas, traz como consequência a diferença entre bom controle e controle deficiente (Montadon, 1993).

Por razões específicas de plantas daninhas problemas, pode ser necessária a adição de mais que um herbicida em sequência de aplicação, para se obter melhor eficiência de controle.

$\mathrm{O}$ objetivo do presente experimento foi avaliar a eficiência de diferentes combinações de tratamentos herbicidas, integrado, pré-plantio incorporado, pré-emergência, pós-emergência, cultivo mecânico e jato dirigido, objetivando colher o algodão no limpo.

\section{MATERIAL E MÉTODOS}

O presente experimento foi instalado no município de Bariri (SP), na Fazenda Bananal, em 
solo classificado como Latossolo Vermelho Escuro, Álico, A moderado, textura argilosa, unidade Limeira (Oliveira et al., 1982; Lepsch \& Oliveira, 1987), com 2,4\% de matéria orgânica e $\mathrm{pH}$ de 5,7.

A área experimental foi instalada na cultura de algodão cultivar IAC-20, plantada em 05 de novembro de 1994. O solo foi previamente preparado com grade Romi, arado de aiveca, grade leve, grade niveladora e plantio. O plantio foi efetuado com plantadeira Semeato - tipo PS-8, com uma densidade de 13/14 sementes/metro linear e profundidade de $3 \mathrm{~cm}$. O espaçamento utilizado foi de $90 \mathrm{~cm}$ entre linhas. A adubação básica por ocasião do plantio foi de $250 \mathrm{~kg} / \mathrm{ha} \mathrm{da}$ fórmula 04.20.20 mais torta de mamona na proporção de 2:1. A cultura recebeu ainda, cobertura de $75 \mathrm{~kg} / \mathrm{ha}$ de uréia.

Foi empregado o delineamento experimental de blocos ao acaso com 12 tratamentos e 3 repetições, compreendendo cada parcela área de $60,0 \times 6,0 \mathrm{~m}$, perfazendo $360,0 \mathrm{~m}^{2}$ de área tratada. Os resultados obtidos foram submetidos à análise de variância (teste F), sendo as médias comparadas pelo teste Tukey a $5 \%$ de probabilidade (Banzatto \& Kronka, 1989).

Os tratamentos foram efetuados em préplantio incorporado (PPI), em pré-emergência (PRE), pós-emergência (POS), em cultivo mecânico (CM) e jato dirigido (JD). A aplicação dos herbicidas em PPI foi realizada em 05/11/1994, no dia do plantio da cultura; em préemergência em 06/11/1994, um dia após o plantio; em pós-emergência em 22/11/1994, 16 dias após o plantio, quando o algodoeiro apresentava 6 folhas e $18 \mathrm{~cm}$ de altura; o cultivo mecânico em 30/12/1994, 55 dias após o plantio, quando o algodoeiro estava com 65 a $70 \mathrm{~cm}$ de altura e o jato dirigido em 19/01/1995, 74 dias após o plantio, com o algodão em fase de préflorescimento. A aplicação dos herbicidas em PPI, PRE e POS foi realizada em área total da parcela, empregando equipamento costal pressurizado com gás carbônico $\left(\mathrm{CO}_{2}\right)$, provido de barra compensada, contendo 4 bicos de jato plano marca TeeJet $\mathrm{T} 110.03$, espaçados entre si de $0,50 \mathrm{~m}$, promovendo $2,0 \mathrm{~m}$ de largura efetiva. O equipamento foi operado a $2,78 \mathrm{Kgf} / \mathrm{cm}^{2}$ (40 psi), empregando água como diluente e volume de aplicação de 220 1/ha. O tratamento em jato dirigido foi efetuado na entre-linha da parcela, empregando o mesmo equipamento provido de uma lança contendo 1 bico defletor marca TeeJet TK VS-2, com protetor tipo chapéu de Napoleão, com largura efetiva de $1,0 \mathrm{~m}$. O equipamento foi operado a $1,67 \mathrm{Kgf} / \mathrm{cm}^{2}$ (25 psi), com volume de aplicação de 450 l/ha.

Foram testados os seguintes herbicidas: trifluralina $445 \mathrm{~g} / \mathrm{l}$ a $1,068 \mathrm{~kg} / \mathrm{ha}$; Laço C.E. (alachlor $480 \mathrm{~g} / \mathrm{l}$ ) a $1,584 \mathrm{~kg} / \mathrm{ha} ;$ Daconate (M.S.M.A. $480 \mathrm{~g} / \mathrm{l}$ ) a 1,44 e $1,68 \mathrm{~kg} / \mathrm{ha}$; Sanson (nicosulfuron $40 \mathrm{~g} / \mathrm{l}$ ) a 0,$020 ; 0,030$ e $0,040 \mathrm{~kg} / \mathrm{ha}$; Karmex (diuron $500 \mathrm{~g} / \mathrm{l}$ ) a 1,0 e $1,5 \mathrm{~kg} / \mathrm{ha}$; Cobra (lactofen $240 \mathrm{~g} / \mathrm{l}$ ) a $0,18 \mathrm{~kg} / \mathrm{ha} ;$ Bladex 500 (cyanazine $500 \mathrm{~g} / \mathrm{l}$ ) a 1,0 e $1,5 \mathrm{~kg} / \mathrm{ha}$ e Cention (diuron $500 \mathrm{~g} / \mathrm{l}$ ) a 1,0 e $1,5 \mathrm{~kg} / \mathrm{ha}$. Os tratamentos avaliados, estão apresentados na Tabela 1.

Para a avaliação dos efeitos dos tratamentos, foram realizadas as seguintes avaliações:

\section{i. Fitotoxicidade Aparente}

A avaliação de fitotoxicidade à cultura foi realizada de forma visual aos $15,30,45$ e 89 D.A.P. (dias após o plantio), empregando a escala visual de 1 a 9 , onde 1 representa "sem sintoma de fitotoxicidade aparente" e o 9 "morte total da planta", conforme a escala EWRC (1964).

\section{ii. Eficiência Agronômica}

As avaliações de Eficiência Agronômica, em função do tipo de cultura e período de competição, foram efetuadas de forma visual aos $15,30,45$ e 89 D.A.P. Em todas as ocasiões foi empregada a escala percentual, onde zero $(0 \%)$ representa nenhum controle e $100 \%$ controle total, comparados à testemunha "sem capina" (ALAM, 1974).

\section{iii. Produção}

A avaliação do rendimento foi efetuada pela colheita, realizada manualmente em 26/04/1995. Para tanto, colheu-se as 4 linhas centrais de cada parcela, desprezando-se os $5,00 \mathrm{~m}$ iniciais e finais como bordaduras. Desta forma, a área colhida representou $144 \mathrm{~m}^{2}$ de cada parcela. $\mathrm{O}$ resultado da colheita foi obtido após a retirada 
das impurezas, pesada e corrigida para a umidade de $13 \%$. Os valores assim obtidos, foram

transformados em $\mathrm{kg} / \mathrm{ha}$ de algodão em caroço.

TABELA 1. Tratamentos químicos e mecânicos utilizados no experimento, com seus respectivos nomes comum e métodos de aplicação (PPI, PRE, POS e JD), e as doses aplicadas em kg i.a/ha.

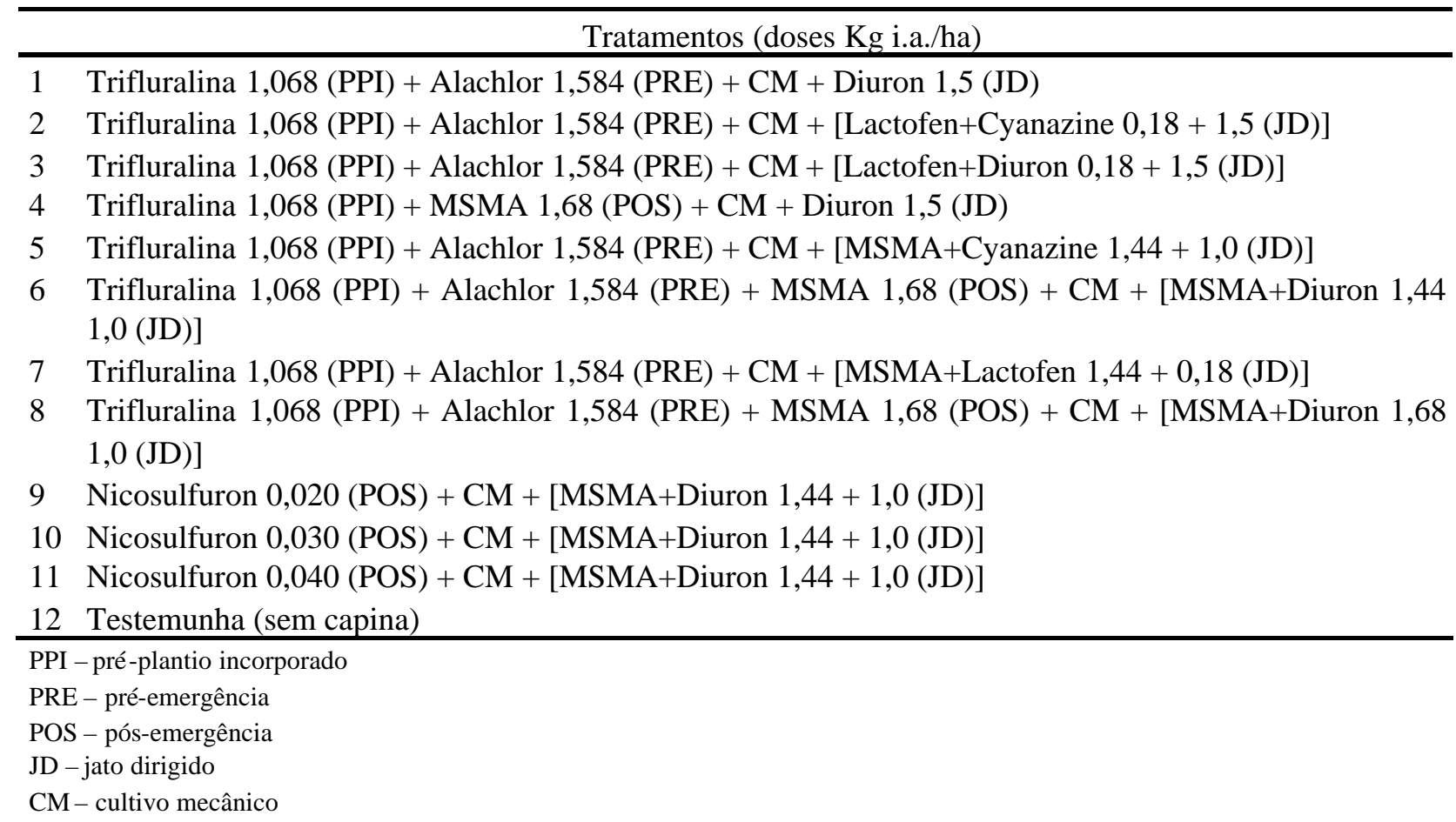

\section{RESULTADOS E DISCUSSÃO}

Os dados sumarizados na Tabela 2 representam os resultados médios relativos à fitotoxicidade aparente, nas avaliações efetuadas aos 15, 30, 45 e 89 D.A.P. Através dessa Tabela, pode-se observar que aos 15 D.A.P. os valores de fitotoxicidade aparente foram elevados, de forte a muito forte, para os tratamentos com nicosulfuron e entre leve e moderada para as aplicações de M.S.M.A. em área total (POS); os demais tratamentos ficaram entre nula e muito leve. Aos 30, 45 e 89 D.A.P., apenas os tratamentos com nicosulfuron mostravam sinais de fitotoxicidade, os quais variaram entre moderada a forte, diminuindo para leve a moderada na última avaliação.

As Tabelas 3 a 9 contém os dados médios relativos ao nível de controle das plantas daninhas, nas avaliações realizadas aos 15, 30, 45 e 89 D.A.P.

Na tabela 3 estão apresentados os resultados referentes a Brachiaria plantaginea e verifica-se que, com exceção dos tratamentos com nicosulfuron nas menores doses, os demais tratamentos proporcionaram excelente nível de controle desta planta daninha. Aos 30 D.A.P. a eficiência era similar, sendo deficiente para as doses de nicosulfuron. Na avaliação de 45 D.A.P. as duas maiores doses de nicosulfuron mostravam alta reinfestação da área. Aos 89 D.A.P. após o cultivo mecânico e aplicação dos herbicidas por jato dirigido, todos os tratamentos mostravam bom nível de controle.

Os dados de controle para Cenchrus echinatus estão contidos na Tabela 4. Os valores dessa Tabela demonstram que todos os tratamentos e doses testadas foram eficientes no 
TABELA 2. Resultados para fitotoxicidade aparente aos 15, 30, 45 e 89 dias após o plantio (D.A.P.) de herbicidas aplicados em PPI (pré-plantio incorporado), PRE (pré-emergência), POS (pós-emergência) e JD (jato dirigido) na cultura do algodão (Bariri - SP, 1994/95).

\begin{tabular}{|c|c|c|c|c|c|}
\hline & Tratamentos & 15 & 30 & 45 & 89 \\
\hline 1 & Trifluralina 1,068 (PPI) + Alachlor 1,584 (PRE) + CM + Diuron 1,5 (JD) & $1,0 \mathrm{c}$ & $1,0 \mathrm{c}$ & $1,0 \mathrm{c}$ & $1,0 \mathrm{~b}$ \\
\hline 2 & Trifluralina 1,068 (PPI) + Alachlor 1,584 (PRE) + CM + [Lactofen+Cyanazine 0,18 + 1,5 (JD)] & $1,0 \mathrm{c}$ & $1,0 \mathrm{c}$ & $1,0 \mathrm{c}$ & $1,0 \mathrm{~b}$ \\
\hline 3 & Trifluralina 1,068 (PPI) + Alachlor 1,584 (PRE) + CM + [Lactofen+Diuron 0,18 + 1,5 (JD)] & $1,3 \mathrm{c}$ & $1,0 \mathrm{c}$ & $1,0 \mathrm{c}$ & $1,0 \mathrm{~b}$ \\
\hline 4 & Trifluralina 1,068 (PPI) + MSMA 1,68 (POS) + CM + Diuron 1,5 (JD) & $4,0 \mathrm{~b}$ & $1,0 \mathrm{c}$ & $1,0 \mathrm{c}$ & $1,0 \mathrm{~b}$ \\
\hline 5 & Trifluralina 1,068 (PPI) + Alachlor 1,584 (PRE) + CM + [MSMA+Cyanazine 1,44 + 1,0 (JD)] & $1,0 \mathrm{c}$ & $1,0 \mathrm{c}$ & $1,0 \mathrm{c}$ & $1,0 \mathrm{~b}$ \\
\hline 6 & $\begin{array}{l}\text { Trifluralina 1,068 (PPI) + Alachlor 1,584 (PRE) + MSMA 1,68 (POS) + CM + [MSMA+Diuron 1,44 - } \\
1,0(\mathrm{JD})]\end{array}$ & $1,0 \mathrm{c}$ & $1,0 \mathrm{c}$ & $1,0 \mathrm{c}$ & $1,0 \mathrm{~b}$ \\
\hline 7 & Trifluralina 1,068 (PPI) + Alachlor 1,584 (PRE) + CM + [MSMA+Lactofen 1,44 + 0,18 (JD)] & $1,0 \mathrm{c}$ & $1,0 \mathrm{c}$ & $1,0 \mathrm{c}$ & $1,0 \mathrm{~b}$ \\
\hline 8 & $\begin{array}{l}\text { Trifluralina 1,068 (PPI) + Alachlor 1,584 (PRE) + MSMA 1,68 (POS) + CM + [MSMA+Diuron 1,68 - } \\
1,0(\mathrm{JD})]\end{array}$ & $3,0 \mathrm{~b}$ & $1,0 \mathrm{c}$ & $1,0 \mathrm{c}$ & $1,0 \mathrm{~b}$ \\
\hline 9 & Nicosulfuron 0,020 (POS) + CM + [MSMA+Diuron 1,44 + 1,0 (JD)] & $6,7 \mathrm{a}$ & $4,3 \mathrm{~b}$ & $3,0 \mathrm{~b}$ & $3,0 \mathrm{a}$ \\
\hline 10 & Nicosulfuron 0,030 (POS) + CM + [MSMA+Diuron 1,44 + 1,0 (JD)] & $7,0 \mathrm{a}$ & $6,0 \mathrm{a}$ & $3,7 \mathrm{~b}$ & $3,0 \mathrm{a}$ \\
\hline 11 & Nicosulfuron 0,040 (POS) + CM + [MSMA+Diuron 1,44 + 1,0 (JD)] & $8,0 \mathrm{a}$ & $7,3 \mathrm{a}$ & $4,7 \mathrm{a}$ & $4,0 \mathrm{a}$ \\
\hline \multirow[t]{4}{*}{12} & Testemunha (sem capina) & $1,0 \mathrm{c}$ & $1,0 \mathrm{c}$ & $1,0 \mathrm{c}$ & $1,0 \mathrm{~b}$ \\
\hline & Tratamentos $(\mathrm{F})$ & $100,91 *$ & $69,67 *$ & $92,14 *$ & $14,09 *$ \\
\hline & C.V. $(\%)$ & 15,71 & 20,45 & 13,91 & 31,58 \\
\hline & d.m.s. $(5 \%)$ & 1,388 & 1,388 & 0,694 & 1,472 \\
\hline
\end{tabular}

* significância ao nível de $1 \%$ de probabilidade.

Médias nas colunas seguidas de mesma letra, não diferem significativamente entre si. 
TABELA 3. Resultados para porcentagem de controle de Brachiaria plantaginea aos 15, 30,45 e 89 dias após o plantio (D.A.P.) de herbicidas aplicados em PPI (pré-plantio incorporado), PRE (pré-emergência), POS (pós-emergência) e JD (jato dirigido) na cultura do algodão (Bariri - SP, 1994/95).

\begin{tabular}{|c|c|c|c|c|c|}
\hline & Tratamentos & 15 & 30 & 45 & 89 \\
\hline 1 & Trifluralina 1,068 (PPI) + Alachlor 1,584 (PRE) + CM + Diuron 1,5 (JD) & $100,0 \mathrm{a}$ & $100,0 \mathrm{a}$ & $100,0 \mathrm{a}$ & 99,7 a \\
\hline 2 & Trifluralina 1,068 (PPI) + Alachlor 1,584 (PRE) + CM + [Lactofen+Cyanazine 0,18 + 1,5 (JD)] & 98,3 a & 97,6 a & 96,6 a & 100,0 a \\
\hline 3 & Trifluralina 1,068 (PPI) + Alachlor 1,584 (PRE) + CM + [Lactofen+Diuron 0,18 + 1,5 (JD)] & $100,0 \mathrm{a}$ & $100,0 \mathrm{a}$ & 93,3 a & $100,0 \mathrm{a}$ \\
\hline 4 & Trifluralina 1,068 (PPI) + MSMA 1,68 (POS) + CM + Diuron 1,5 (JD) & 100,0 a & 100,0 a & 100,0 a & 100,0 a \\
\hline 5 & Trifluralina 1,068 (PPI) + Alachlor 1,584 (PRE) + CM + [MSMA+Cyanazine 1,44 + 1,0 (JD)] & 100,0 a & 99,3 a & 93,3 a & 99,3 a \\
\hline 6 & $\begin{array}{l}\text { Trifluralina 1,068 (PPI) + Alachlor 1,584 (PRE) + MSMA 1,68 (POS) + CM + [MSMA+Diuron 1,44 } \\
1,0(\mathrm{JD})]\end{array}$ & $100,0 \mathrm{a}$ & $100,0 \mathrm{a}$ & 91,7 a & $100,0 \mathrm{a}$ \\
\hline 7 & Trifluralina 1,068 (PPI) + Alachlor 1,584 (PRE) + CM + [MSMA+Lactofen 1,44 + 0,18 (JD)] & 100,0 a & 96,7 a & 91,7 a & 99,3 a \\
\hline 8 & $\begin{array}{l}\text { Trifluralina 1,068 (PPI) + Alachlor 1,584 (PRE) + MSMA 1,68 (POS) + CM + [MSMA+Diuron 1,68 } \\
1,0(\text { JD)] }\end{array}$ & $100,0 \mathrm{a}$ & 99,7 a & 96,7 a & $100,0 \mathrm{a}$ \\
\hline 9 & Nicosulfuron 0,020 (POS) + CM + [MSMA+Diuron 1,44 + 1,0 (JD)] & $81,7 \mathrm{c}$ & $66,7 \mathrm{c}$ & $21,0 \mathrm{a}$ & $88,3 \mathrm{~b}$ \\
\hline 10 & Nicosulfuron $0,030(\mathrm{POS})+\mathrm{CM}+[\mathrm{MSMA}+$ Diuron 1,44 + 1,0 (JD)] & $91,7 \mathrm{~b}$ & $80,0 \mathrm{~b}$ & $40,0 \mathrm{~b}$ & $100,0 \mathrm{a}$ \\
\hline 11 & Nicosulfuron $0,040(\mathrm{POS})+\mathrm{CM}+[\mathrm{MSMA}+$ Diuron 1,44 + 1,0 (JD)] & $96,0 \mathrm{ab}$ & $80,0 \mathrm{~b}$ & $90,0 \mathrm{~b}$ & $91,7 \mathrm{~b}$ \\
\hline \multirow[t]{4}{*}{12} & Testemunha (sem capina) & $0,0 \mathrm{~d}$ & $0,0 \mathrm{~d}$ & $0,0 \mathrm{c}$ & $0,0 \mathrm{c}$ \\
\hline & Tratamentos $(\mathrm{F})$ & $1047,86^{* *}$ & $608,08 *$ & $208,26^{*}$ & $421,52 *$ \\
\hline & C.V. $(\%)$ & 1,72 & 2,39 & 7,38 & 2,68 \\
\hline & d.m.s. $(5 \%)$ & 4,498 & 5,990 & 14,722 & 7,095 \\
\hline
\end{tabular}

* significância ao nivel de $1 \%$ de probabilidade.

Médias nas colunas seguidas de mesma letra, não diferem significativamente entre si. 
TABELA 4. Resultados para porcentagem de controle de Cenchrus echinatus aos 15, 30, 45 e 89 dias após o plantio (D.A.P.) de herbicidas aplicados em PPI (pré-plantio incorporado), PRE (pré-emergência), POS (pós-emergência) e JD (jato dirigido) na cultura do algodão (Bariri - SP, 1994/95).

\begin{tabular}{|c|c|c|c|c|c|}
\hline & Tratamentos & 15 & 30 & 45 & 89 \\
\hline 1 & Trifluralina 1,068 (PPI) + Alachlor 1,584 (PRE) + CM + Diuron 1,5 (JD) & $100,0 \mathrm{a}$ & $100,0 \mathrm{a}$ & $100,0 \mathrm{a}$ & $100,0 \mathrm{a}$ \\
\hline 2 & Trifluralina 1,068 (PPI) + Alachlor 1,584 (PRE) $+\mathrm{CM}+[$ Lactofen+Cyanazine 0,18 + 1,5 (JD)] & 96,7 a & 96,0 a & 98,3 a & $100,0 \mathrm{a}$ \\
\hline 3 & Trifluralina 1,068 (PPI) + Alachlor 1,584 (PRE) + CM + [Lactofen+Diuron 0,18 + 1,5 (JD)] & $100,0 \mathrm{a}$ & $100,0 \mathrm{a}$ & $100,0 \mathrm{a}$ & $100,0 \mathrm{a}$ \\
\hline 4 & Trifluralina 1,068 (PPI) + MSMA 1,68 (POS) + CM + Diuron 1,5 (JD) & $100,0 \mathrm{a}$ & $100,0 \mathrm{a}$ & $100,0 \mathrm{a}$ & $100,0 \mathrm{a}$ \\
\hline 5 & Trifluralina 1,068 (PPI) + Alachlor 1,584 (PRE) + CM + [MSMA+Cyanazine 1,44 + 1,0 (JD)] & $100,0 \mathrm{a}$ & $100,0 \mathrm{a}$ & $100,0 \mathrm{a}$ & $100,0 \mathrm{a}$ \\
\hline 6 & $\begin{array}{l}\text { Trifluralina 1,068 (PPI) + Alachlor 1,584 (PRE) + MSMA 1,68 (POS) + CM + [MSMA+Diuron 1,44 } \\
1,0(\mathrm{JD})]\end{array}$ & $100,0 \mathrm{a}$ & $100,0 \mathrm{a}$ & $100,0 \mathrm{a}$ & $100,0 \mathrm{a}$ \\
\hline 7 & Trifluralina 1,068 (PPI) + Alachlor 1,584 (PRE) + CM + [MSMA+Lactofen 1,44 + 0,18 (JD)] & 100,0 a & $100,0 \mathrm{a}$ & $100,0 \mathrm{a}$ & 100,0 a \\
\hline 8 & $\begin{array}{l}\text { Trifluralina 1,068 (PPI) + Alachlor 1,584 (PRE) + MSMA 1,68 (POS) + CM + [MSMA+Diuron 1,68 } \\
1,0(\mathrm{JD})]\end{array}$ & 100,0 a & $100,0 \mathrm{a}$ & 100,0 a & $100,0 \mathrm{a}$ \\
\hline 9 & Nicosulfuron $0,020(\mathrm{POS})+\mathrm{CM}+[\mathrm{MSMA}+$ Diuron $1,44+1,0(\mathrm{JD})]$ & $95,0 \mathrm{~b}$ & 96,7 a & $80,0 \mathrm{c}$ & $97,3 \mathrm{~b}$ \\
\hline 10 & Nicosulfuron 0,030 (POS) + CM + [MSMA+Diuron 1,44 + 1,0 (JD)] & $100,0 \mathrm{a}$ & 99,3 a & $93,3 \mathrm{~b}$ & $100,0 \mathrm{a}$ \\
\hline 11 & Nicosulfuron 0,040 (POS) + CM + [MSMA+Diuron 1,44 + 1,0 (JD)] & 100,0 a & $100,0 \mathrm{a}$ & $95,0 \mathrm{~b}$ & $100,0 \mathrm{a}$ \\
\hline \multirow[t]{4}{*}{12} & Testemunha (sem capina) & $0,0 \mathrm{c}$ & $0,0 \mathrm{~b}$ & $0,0 \mathrm{c}$ & $0,0 \mathrm{c}$ \\
\hline & Tratamentos $(\mathrm{F})$ & $889,34 *$ & $472,90^{*}$ & $581,22 *$ & $6894,43^{* * *}$ \\
\hline & C.V. $(\%)$ & 1,83 & 2,51 & 3,36 & 0,66 \\
\hline & d.m.s. $(5 \%)$ & 4,907 & 6,728 & 8,138 & 1,769 \\
\hline
\end{tabular}

* significância ao nivel de $1 \%$ de probabilidade

Médias nas colunas seguidas de mesma letra, não diferem significativamente entre si. 
controle dessa espécie aos 15, 30, 45 D.A.P.; após o cultivo mecânico e aplicação dos herbicidas em jato dirigido (89 D.A.P.).

Os dados médios da Tabela 5 mostram para Commelina benghalensis que todos os produtos e doses foram eficientes no controle dessa espécie aos 15 D.A.P., exceto as duas menores doses de nicosulfuron. Aos 30 D.A.P. observou-se reinfestação por esta espécie daninha, nas áreas tratadas com nicosulfuron + M.S.M.A. + diuron em todas as dosagens utilizadas. Aos 45 D.A.P. apenas os dois primeiros tratamentos mantinham bom nível de controle. Aos 89 D.A.P. apenas o tratamento 9 (nicosulfuron $0,020 \mathrm{~kg}$ i.a./ha (POS) $+\mathrm{CM}+$ [M.S.M.A. + Diuron a $1,44+1,0 \mathrm{~kg}$ i.a./ha] (JD)) não mostrou bom nível de controle, tendo em vista o alto nível de reinfestação observada antes da aplicação do jato dirigido.

$\mathrm{Na}$ Tabela 6 estão apresentados os resultados obtidos para o controle de Sida cordifolia, pela qual pode-se observar que esta espécie é suscetível aos tratamentos efetuados na avaliação de 15 D.A.P.. Aos 30 D.A.P. observouse reinfestação para os tratamentos 3 (Trifluralina PPI + Alachlor PRE + CM + Lactofen + Diuron JD) , 5 (Trifluralina PPI + Alachlor PRE + CM + M.S.M.A. + Cyanazine JD), 7 (Trifluralina PPI + Alachlor PRE + CM + M.S.M.A. + Lactofen JD) e 8 (Trifluralina PPI + Alachlor PRE + M.S.M.A. POS + CM + M.S.M.A. + Diuron JD), sendo que os demais apresentavam bom nível de controle. Aos 45 D.A.T. apenas os tratamentos 1 (Trifluralina PPI + Alachlor PRE + CM + Diuron JD), 4 (Trifluralina PPI + M.S.M.A. POS + CM + Diuron JD) e 6 (Trifluralina PPI + Alachlor PRE + M.S.M.A. POS + CM + M.S.M.A. + Diuron JD) mantinham controle superior à $80 \%$. Após a aplicação dos herbicidas por jato dirigido, todos os tratamentos mostravam bom nível de controle, exceto as duas menores doses de nicosulfuron, devido ao alto grau de infestação anterior ao tratamento.

Conforme os dados da Tabela 7, pode-se verificar que o nível de controle de Ipomoea grandifolia variou de bom a excelente para todos os tratamentos aos 15 D.A.P.. Aos 30 D.A.P. apenas o tratamento 7 (Trifluralina PPI + Alachlor PRE + CM + M.S.M.A. + Lactofen JD) mostrava reinfestação. Aos 45 D.A.P. a maioria dos tratamentos mostravam bom nível de controle. Aos 89 D.A.P. todos os tratamentos mostravam alta eficiência de controle.

Conforme pode ser observado na Tabela 8 , aos 15 D.A.P. vários tratamentos foram eficientes no controle de Acanthospermum hispidum. Já aos 30 D.A.P. a eficiência era observada apenas para os tratamentos 4 (Trifluralina PPI + M.S.M.A. POS + CM + Diuron JD) e os tratamentos com as duas maiores dosagens de nicosulfuron (10 e 11). Aos 45 D.A.P. a eficiência foi observada, apenas para os tratamentos 4 e 11 . Após o tratamento de cultivo mecânico e jato dirigido todos os tratamentos mostraram-se eficientes.

Bidens pilosa foi controlada aos 15 D.A.P. por todos os tratamentos estudados, e aos 30 D.A.P. apenas os tratamentos 4 (Trifluralina PPI + M.S.M.A. POS + CM + Diuron JD) e aqueles onde foi realizada a aplicação em pós-emergência de nicosulfuron nas três dosagens empregadas $(9,10$ e 11) mostravam-se eficientes, sendo os 3 últimos $100 \%$ eficientes e aos 45 D.A.P. os tratamentos 4 , 5 (Trifluralina PPI + Alachlor PRE + CM + M.S.M.A. + Cyanazine JD) e as duas dosagens mais altas de nicosulfuron aplicados em pósemergência (10 e 11) controlaram eficientemente esta espécie. Aos 89 D.A.P. todos os tratamentos mostravam bom nível de controle (Tabela 9).

A Tabela 10 apresenta os dados relativos à produção em $\mathrm{kg} / \mathrm{ha}$ de algodão em caroço, pela qual pode-se observar que o tratamento 7 mostrou maior produção de $2638,9 \mathrm{~kg} / \mathrm{ha}$, seguida pelo tratamento 6. Os tratamentos $1,3,5$ e 6 não diferiram significativamente destes. As menores produções foram para os tratamentos 9,10 e 11, resultado da aplicação do nicosulfuron com alta fitotoxicidade, a qual prejudica o desenvolvimento normal da cultura. A testemunha produziu apenas $427,6 \mathrm{~kg} / \mathrm{ha}$.

Através dos resultados obtidos pode-se verificar que os sistemas herbicidas utilizados provocaram efeito de fitotoxicidade aparente entre nula (diversos tratamentos), leve a moderada para 
TABELA 5. Resultados para porcentagem de controle de Commelina bengalensis aos 15, 30, 45 e 89 dias após o plantio (D.A.P.) de herbicidas aplicados em PPI (pré-plantio incorporado), PRE (pré-emergência), POS (pós-emergência) e JD (jato dirigido) na cultura do algodão (Bariri - SP, 1994/95).

\begin{tabular}{|c|c|c|c|c|c|}
\hline & Tratamentos & 15 & 30 & 45 & 89 \\
\hline 1 & Trifluralina 1,068 (PPI) + Alachlor 1,584 (PRE) + CM + Diuron 1,5 (JD) & $83,3 \mathrm{a}$ & $88,3 \mathrm{ab}$ & $88,3 \mathrm{a}$ & $100,0 \mathrm{a}$ \\
\hline 2 & Trifluralina 1,068 (PPI) + Alachlor 1,584 (PRE) + CM + [Lactofen+Cyanazine 0,18 + 1,5 (JD)] & 91,7 a & 91,7 a & 88,3 a & $100,0 \mathrm{a}$ \\
\hline 3 & Trifluralina 1,068 (PPI) + Alachlor 1,584 (PRE) + CM + [Lactofen+Diuron 0,18 + 1,5 (JD)] & 88,3 a & $50,0 \mathrm{c}$ & $56,7 \mathrm{~b}$ & 96,0 a \\
\hline 4 & Trifluralina 1,068 (PPI) + MSMA 1,68 (POS) + CM + Diuron 1,5 (JD) & 96,7 a & $78,3 \mathrm{abc}$ & $76,7 \mathrm{ab}$ & $100,0 \mathrm{a}$ \\
\hline 5 & Trifluralina 1,068 (PPI) + Alachlor 1,584 (PRE) + CM + [MSMA+Cyanazine 1,44 + 1,0 (JD)] & 92,7 a & $80,0 \mathrm{abc}$ & $76,7 \mathrm{ab}$ & 98,3 a \\
\hline 6 & $\begin{array}{l}\text { Trifluralina 1,068 (PPI) + Alachlor 1,584 (PRE) + MSMA 1,68 (POS) + CM + [MSMA+Diuron 1,44 } \\
1,0(\mathrm{JD})]\end{array}$ & $95,0 \mathrm{a}$ & $81,7 \mathrm{abc}$ & $71,7 \mathrm{ab}$ & 99,7 a \\
\hline 7 & Trifluralina 1,068 (PPI) + Alachlor 1,584 (PRE) + CM + [MSMA+Lactofen 1,44 + 0,18 (JD)] & 83,3 a & $55,0 \mathrm{c}$ & $53,3 \mathrm{~b}$ & 99,0 a \\
\hline 8 & $\begin{array}{l}\text { Trifluralina 1,068 (PPI) + Alachlor 1,584 (PRE) + MSMA 1,68 (POS) + CM + [MSMA+Diuron 1,68 } \\
1,0(\mathrm{JD})]\end{array}$ & 88,3 a & $76,7 \mathrm{abc}$ & $53,3 \mathrm{~b}$ & 99,7 a \\
\hline 9 & Nicosulfuron $0,020(\mathrm{POS})+\mathrm{CM}+[\mathrm{MSMA}+$ Diuron $1,44+1,0(\mathrm{JD})]$ & $13,3 \mathrm{c}$ & $0,0 \mathrm{~d}$ & $0,0 \mathrm{c}$ & $53,3 \mathrm{c}$ \\
\hline 10 & Nicosulfuron 0,030 (POS) + CM + [MSMA+Diuron 1,44 + 1,0 (JD)] & $46,7 \mathrm{~b}$ & $60,0 \mathrm{bc}$ & $16,6 \mathrm{c}$ & $85,0 \mathrm{~b}$ \\
\hline 11 & Nicosulfuron 0,040 (POS) + CM + [MSMA+Diuron 1,44 + 1,0 (JD)] & 83,3 a & $75,0 \mathrm{abc}$ & $53,3 \mathrm{~b}$ & 93,3 a \\
\hline \multirow[t]{4}{*}{12} & Testemunha (sem capina) & $0,0 \mathrm{c}$ & $0,0 \mathrm{~d}$ & $0,0 \mathrm{c}$ & $0,0 \mathrm{~d}$ \\
\hline & Tratamentos $(\mathrm{F})$ & $161,26^{*}$ & $31,23^{*}$ & $37,26^{*}$ & $406,52^{*}$ \\
\hline & C.V. $(\%)$ & 6,30 & 17,62 & 16,89 & 3,02 \\
\hline & d.m.s. $(5 \%)$ & 13,341 & 30,549 & 26,313 & 7,587 \\
\hline
\end{tabular}

* significância ao nivel de $1 \%$ de probabilidade

Médias nas colunas seguidas de mesma letra, não diferem significativamente entre si. 
TABELA 6. Resultados obtidos para porcentagem de controle de Sida cordifolia aos 15, 30 , 45 e 89 dias após o plantio (D.A.P.) de herbicidas aplicados em PPI (pré-plantio incorporado), PRE (pré-emergência), POS (pós-emergência) e JD (jato dirigido) na cultura do algodão (Bariri - SP, 1994/95).

\begin{tabular}{|c|c|c|c|c|c|}
\hline & Tratamentos & 15 & 30 & 45 & 89 \\
\hline 1 & Trifluralina 1,068 (PPI) + Alachlor 1,584 (PRE) + CM + Diuron 1,5 (JD) & $95,0 \mathrm{a}$ & $91,7 \mathrm{a}$ & 83,3 a & $100,0 \mathrm{a}$ \\
\hline 2 & Trifluralina 1,068 (PPI) + Alachlor 1,584 (PRE) + CM + [Lactofen+Cyanazine 0,18 + 1,5 (JD)] & 83,0 a & 88,3 a & $73,3 \mathrm{abc}$ & $100,0 \mathrm{a}$ \\
\hline 3 & Trifluralina 1,068 (PPI) + Alachlor 1,584 (PRE) + CM + [Lactofen+Diuron 0,18 + 1,5 (JD)] & $90,0 \mathrm{a}$ & $63,3 \mathrm{~b}$ & $60,0 \mathrm{bc}$ & $100,0 \mathrm{a}$ \\
\hline 4 & Trifluralina 1,068 (PPI) + MSMA 1,68 (POS) + CM + Diuron 1,5 (JD) & 86,7 a & 83,3 a & 83,3 a & $100,0 \mathrm{a}$ \\
\hline 5 & Trifluralina 1,068 (PPI) + Alachlor 1,584 (PRE) + CM + [MSMA+Cyanazine 1,44 + 1,0 (JD)] & $96,0 \mathrm{a}$ & $68,3 \mathrm{a}$ & $50,0 \mathrm{~d}$ & $100,0 \mathrm{a}$ \\
\hline 6 & $\begin{array}{l}\text { Trifluralina 1,068 (PPI) + Alachlor 1,584 (PRE) + MSMA 1,68 (POS) + CM + [MSMA+Diuron 1,44 } \\
1,0(\mathrm{JD})]\end{array}$ & 96,7 a & 88,3 a & $81,7 \mathrm{ab}$ & $100,0 \mathrm{a}$ \\
\hline 7 & Trifluralina 1,068 (PPI) + Alachlor 1,584 (PRE) + CM + [MSMA+Lactofen 1,44 + 0,18 (JD)] & $91,7 \mathrm{a}$ & $65,0 \mathrm{a}$ & $56,7 \mathrm{c}$ & $100,0 \mathrm{a}$ \\
\hline 8 & $\begin{array}{l}\text { Trifluralina 1,068 (PPI) + Alachlor 1,584 (PRE) + MSMA 1,68 (POS) + CM + [MSMA+Diuron 1,68 } \\
1,0(\mathrm{JD})]\end{array}$ & 83,3 a & $73,3 \mathrm{a}$ & $63,3 \mathrm{abc}$ & $100,0 \mathrm{a}$ \\
\hline 9 & Nicosulfuron 0,020 (POS) + CM + [MSMA+Diuron 1,44 + 1,0 (JD)] & $95,0 \mathrm{a}$ & 83,3 a & $75,0 \mathrm{abc}$ & $73,3 \mathrm{~b}$ \\
\hline 10 & Nicosulfuron 0,030 (POS) + CM + [MSMA+Diuron 1,44 + 1,0 (JD)] & $96,0 \mathrm{a}$ & $85,0 \mathrm{a}$ & $76,7 \mathrm{abc}$ & $78,3 \mathrm{~b}$ \\
\hline 11 & Nicosulfuron 0,040 (POS) + CM + [MSMA+Diuron 1,44 + 1,0 (JD)] & $97,0 \mathrm{a}$ & 88,3 a & $78,3 \mathrm{abc}$ & $81,7 \mathrm{~b}$ \\
\hline \multirow[t]{4}{*}{12} & Testemunha (sem capina) & $0,0 \mathrm{~b}$ & $0,0 \mathrm{c}$ & $0,0 \mathrm{e}$ & $0,0 \mathrm{c}$ \\
\hline & Tratamentos $(\mathrm{F})$ & $48,38^{*}$ & $25,32 *$ & $43,18^{*}$ & $100,38^{*}$ \\
\hline & C.V. $(\%)$ & 7,97 & 14,18 & 12,89 & 5,81 \\
\hline & d.m.s. $(5 \%)$ & 19,795 & 28,825 & 23,148 & 14,722 \\
\hline
\end{tabular}

* significância ao nivel de $1 \%$ de probabilidade

Médias nas colunas seguidas de mesma letra, não diferem significativamente entre si. 
TABELA 7. Resultados para porcentagem de controle de Ipomoea grandiflora aos 15, 30 , 45 e 89 dias após o plantio (D.A.P.) de herbicidas aplicados em PPI (pré-plantio incorporado), PRE (pré-emergência), POS (pós-emergência) e JD (jato dirigido) na cultura do algodão (Bariri - SP, 1994/95).

\begin{tabular}{|c|c|c|c|c|c|}
\hline & Tratamentos & 15 & 30 & 45 & 89 \\
\hline 1 & Trifluralina 1,068 (PPI) + Alachlor 1,584 (PRE) + CM + Diuron 1,5 (JD) & $86,7 \mathrm{ab}$ & $92,7 \mathrm{a}$ & $63,3 \mathrm{bcd}$ & 100,0 a \\
\hline 2 & Trifluralina 1,068 (PPI) + Alachlor 1,584 (PRE) + CM + [Lactofen+Cyanazine 0,18 + 1,5 (JD)] & $93,3 \mathrm{ab}$ & 88,3 a & $63,3 \mathrm{bcd}$ & $100,0 \mathrm{a}$ \\
\hline 3 & Trifluralina 1,068 (PPI) + Alachlor 1,584 (PRE) + CM + [Lactofen+Diuron 0,18 + 1,5 (JD)] & $85,0 \mathrm{ab}$ & $51,7 \mathrm{~cd}$ & $60,0 \mathrm{~cd}$ & $98,3 \mathrm{a}$ \\
\hline 4 & Trifluralina 1,068 (PPI) + MSMA 1,68 (POS) + CM + Diuron 1,5 (JD) & $100,0 \mathrm{a}$ & $92,7 \mathrm{a}$ & $95,0 \mathrm{a}$ & 100,0 a \\
\hline 5 & Trifluralina 1,068 (PPI) + Alachlor 1,584 (PRE) + CM + [MSMA+Cyanazine 1,44 + 1,0 (JD)] & 98,3 a & 88,3 a & $85,0 \mathrm{abc}$ & $100,0 \mathrm{a}$ \\
\hline 6 & $\begin{array}{l}\text { Trifluralina 1,068 (PPI) + Alachlor 1,584 (PRE) + MSMA 1,68 (POS) + CM + [MSMA+Diuron 1,44 } \\
1,0(\mathrm{JD})]\end{array}$ & $94,3 \mathrm{ab}$ & 86,7 a & $86,6 \mathrm{abc}$ & 100,0 a \\
\hline 7 & Trifluralina 1,068 (PPI) + Alachlor 1,584 (PRE) + CM + [MSMA+Lactofen 1,44 + 0,18 (JD)] & $80,0 \mathrm{~b}$ & $63,3 \mathrm{bc}$ & $50,0 \mathrm{~d}$ & 99,3 a \\
\hline 8 & $\begin{array}{l}\text { Trifluralina 1,068 (PPI) + Alachlor 1,584 (PRE) + MSMA 1,68 (POS) + CM + [MSMA+Diuron 1,68 } \\
1,0(\mathrm{JD})]\end{array}$ & $91,7 \mathrm{ab}$ & 93,3 a & 97,6 a & 98,7 a \\
\hline 9 & Nicosulfuron $0,020(\mathrm{POS})+\mathrm{CM}+[\mathrm{MSMA}+$ Diuron $1,44+1,0(\mathrm{JD})]$ & $91,7 \mathrm{ab}$ & $85,0 \mathrm{ab}$ & $40,0 \mathrm{~d}$ & $90,0 \mathrm{~b}$ \\
\hline 10 & Nicosulfuron 0,030 (POS) + CM + [MSMA+Diuron 1,44 + 1,0 (JD)] & $95,0 \mathrm{ab}$ & 88,3 a & $90,0 \mathrm{abc}$ & 99,3 a \\
\hline 11 & Nicosulfuron 0,040 (POS) + CM + [MSMA+Diuron 1,44 + 1,0 (JD)] & $95,0 \mathrm{ab}$ & 90,0 a & $91,7 \mathrm{ab}$ & 99,3 a \\
\hline \multirow[t]{4}{*}{12} & Testemunha (sem capina) & $0,0 \mathrm{c}$ & $0,0 \mathrm{~d}$ & $0,0 \mathrm{e}$ & $0,0 \mathrm{c}$ \\
\hline & Tratamentos $(\mathrm{F})$ & $69,93 *$ & $24,22 *$ & $30,88^{*}$ & $2330,84 * *$ \\
\hline & C.V. $(\%)$ & 6,92 & 15,05 & 16,35 & 1,14 \\
\hline & d.m.s. $(5 \%)$ & 17,169 & 32,419 & 31,400 & 3,025 \\
\hline
\end{tabular}

* significância ao nivel de $1 \%$ de probabilidade

Médias nas colunas seguidas de mesma letra, não diferem significativamente entre si. 
TABELA 8. Resultados obtidos porcentagem de controle de Acanthopermum hispidum aos 15, 30, 45 e 89 dias após o plantio (D.A.P.) de herbicidas aplicados em PPI (pré-plantio incorporado), PRE (pré-emergência), POS (pós-emergência) e JD (jato dirigido) na cultura do algodão (Bariri - SP, 1994/95).

\begin{tabular}{|c|c|c|c|c|c|}
\hline & Tratamentos & 15 & 30 & 45 & 89 \\
\hline 1 & Trifluralina 1,068 (PPI) + Alachlor 1,584 (PRE) + CM + Diuron 1,5 (JD) & $80,0 \mathrm{ab}$ & $52,7 \mathrm{~cd}$ & $36,7 \mathrm{~b}$ & $100,0 \mathrm{a}$ \\
\hline 2 & Trifluralina 1,068 (PPI) + Alachlor 1,584 (PRE) $+\mathrm{CM}+[$ Lactofen+Cyanazine $0,18+1,5(\mathrm{JD})]$ & $76,7 \mathrm{ab}$ & $36,7 \mathrm{~d}$ & $23,3 \mathrm{bc}$ & $100,0 \mathrm{a}$ \\
\hline 3 & Trifluralina 1,068 (PPI) + Alachlor 1,584 (PRE) $+\mathrm{CM}+[$ Lactofen+Diuron $0,18+1,5(\mathrm{JD})]$ & $73,3 \mathrm{~b}$ & 13,3 ef & $16,7 \mathrm{bc}$ & $100,0 \mathrm{a}$ \\
\hline 4 & Trifluralina 1,068 (PPI) + MSMA 1,68 (POS) + CM + Diuron 1,5 (JD) & $95,0 \mathrm{a}$ & $80,0 \mathrm{ab}$ & $80,0 \mathrm{a}$ & 99,3 a \\
\hline 5 & Trifluralina 1,068 (PPI) + Alachlor 1,584 (PRE) + CM + [MSMA+Cyanazine 1,44 + 1,0 (JD)] & $93,3 \mathrm{ab}$ & $60,0 \mathrm{bc}$ & $70,0 \mathrm{a}$ & $100,0 \mathrm{a}$ \\
\hline 6 & $\begin{array}{l}\text { Trifluralina 1,068 (PPI) + Alachlor 1,584 (PRE) + MSMA 1,68 (POS) + CM + [MSMA+Diuron 1,44 } \\
1,0(\mathrm{JD})]\end{array}$ & $81,7 \mathrm{ab}$ & $53,3 \mathrm{~cd}$ & $73,3 \mathrm{a}$ & 99,7 a \\
\hline 7 & Trifluralina 1,068 (PPI) + Alachlor 1,584 (PRE) + CM + [MSMA+Lactofen 1,44 + 0,18 (JD)] & $76,7 \mathrm{ab}$ & $33,3 \mathrm{de}$ & $20,0 \mathrm{bc}$ & $98,3 \mathrm{a}$ \\
\hline 8 & $\begin{array}{l}\text { Trifluralina 1,068 (PPI) + Alachlor 1,584 (PRE) + MSMA 1,68 (POS) + CM + [MSMA+Diuron 1,68 } \\
1,0(\mathrm{JD})]\end{array}$ & $90,0 \mathrm{ab}$ & $50,0 \mathrm{~cd}$ & $70,0 \mathrm{a}$ & $100,0 \mathrm{a}$ \\
\hline 9 & Nicosulfuron $0,020(\mathrm{POS})+\mathrm{CM}+[$ MSMA+Diuron 1,44 + 1,0 (JD) $]$ & $75,0 \mathrm{ab}$ & $70,0 \mathrm{abc}$ & $20,0 \mathrm{bc}$ & $83,3 \mathrm{~b}$ \\
\hline 10 & Nicosulfuron $0,030(\mathrm{POS})+\mathrm{CM}+[\mathrm{MSMA}+$ Diuron $1,44+1,0(\mathrm{JD})]$ & $81,7 \mathrm{ab}$ & $81,7 \mathrm{a}$ & $76,7 \mathrm{a}$ & $88,3 \mathrm{~b}$ \\
\hline 11 & Nicosulfuron $0,040(\mathrm{POS})+\mathrm{CM}+[$ MSMA+Diuron 1,44 + 1,0 (JD)] & $83,3 \mathrm{ab}$ & $88,3 \mathrm{a}$ & $80,0 \mathrm{a}$ & $80,0 \mathrm{~b}$ \\
\hline \multirow[t]{4}{*}{12} & Testemunha (sem capina) & $0,0 \mathrm{c}$ & $0,0 \mathrm{f}$ & $0,0 \mathrm{c}$ & $0,0 \mathrm{c}$ \\
\hline & Tratamentos $(\mathrm{F})$ & $38,57^{*}$ & $42,98^{*}$ & $29,76^{*}$ & $287,05^{*}$ \\
\hline & C.V. $(\%)$ & 9,18 & 13,94 & 22,55 & 3,33 \\
\hline & d.m.s. $(5 \%)$ & 20,529 & 21,187 & 30,252 & 8,507 \\
\hline
\end{tabular}

* significância ao nivel de $1 \%$ de probabilidade

Médias nas colunas seguidas de mesma letra, não diferem significativamente entre si. 
TABELA 9. Resultados obtidos porcentagem de controle de Bidens pilosa aos 15, 30, 45 e 89 dias após o plantio (D.A.P.) de herbicidas aplicados em PPI (pré-plantio incorporado), PRE (pré-emergência), POS (pós-emergência) e JD (jato dirigido) na cultura do algodão (Bariri - SP, 1994/95).

\begin{tabular}{|c|c|c|c|c|c|}
\hline & Tratamentos & 15 & 30 & 45 & 89 \\
\hline 1 & Trifluralina 1,068 (PPI) + Alachlor 1,584 (PRE) + CM + Diuron 1,5 (JD) & $80,0 \mathrm{bc}$ & $50,0 \mathrm{bcd}$ & $56,7 \mathrm{~cd}$ & $100,0 \mathrm{a}$ \\
\hline 2 & Trifluralina 1,068 (PPI) + Alachlor 1,584 (PRE) + CM + [Lactofen+Cyanazine 0,18 + 1,5 (JD)] & $76,7 \mathrm{c}$ & 36,7 cde & $50,0 \mathrm{~d}$ & $100,0 \mathrm{a}$ \\
\hline 3 & Trifluralina 1,068 (PPI) + Alachlor 1,584 (PRE) + CM + [Lactofen+Diuron 0,18 + 1,5 (JD)] & $73,3 \mathrm{c}$ & $13,3 \mathrm{~cd}$ & $50,0 \mathrm{~d}$ & $100,0 \mathrm{a}$ \\
\hline 4 & Trifluralina 1,068 (PPI) + MSMA 1,68 (POS) + CM + Diuron 1,5 (JD) & $95,0 \mathrm{ab}$ & $85,0 \mathrm{ab}$ & $80,0 \mathrm{abc}$ & 98,3 a \\
\hline 5 & Trifluralina 1,068 (PPI) + Alachlor 1,584 (PRE) + CM + [MSMA+Cyanazine 1,44 + 1,0 (JD)] & $93,3 \mathrm{ab}$ & $71,7 \mathrm{abc}$ & $80,0 \mathrm{abc}$ & $100,0 \mathrm{a}$ \\
\hline 6 & $\begin{array}{l}\text { Trifluralina 1,068 (PPI) + Alachlor 1,584 (PRE) + MSMA 1,68 (POS) + CM + [MSMA+Diuron } \\
1,44+1,0(\mathrm{JD})]\end{array}$ & $81,7 \mathrm{bc}$ & $63,3 \mathrm{abc}$ & 75,0 abcd & 99,7 a \\
\hline 7 & Trifluralina 1,068 (PPI) + Alachlor 1,584 (PRE) + CM + [MSMA+Lactofen 1,44 + 0,18 (JD)] & $76,7 \mathrm{c}$ & $50,0 \mathrm{bcd}$ & $53,3 \mathrm{~d}$ & 97,7 a \\
\hline 8 & $\begin{array}{l}\text { Trifluralina 1,068 (PPI) + Alachlor 1,584 (PRE) + MSMA 1,68 (POS) + CM + [MSMA+Diuron } \\
1,68+1,0(\mathrm{JD})]\end{array}$ & $85,0 \mathrm{abc}$ & $65,0 \mathrm{abc}$ & $73,3 \mathrm{bcd}$ & $100,0 \mathrm{a}$ \\
\hline 9 & Nicosulfuron 0,020 (POS) + CM + [MSMA+Diuron 1,44 + 1,0 (JD)] & 98,3 a & $100,0 \mathrm{a}$ & $50,0 \mathrm{~d}$ & $90,0 \mathrm{~b}$ \\
\hline 10 & Nicosulfuron $0,030(\mathrm{POS})+\mathrm{CM}+[\mathrm{MSMA}+$ Diuron $1,44+1,0(\mathrm{JD})]$ & $100,0 \mathrm{a}$ & $100,0 \mathrm{a}$ & $95,0 \mathrm{ab}$ & $100,0 \mathrm{a}$ \\
\hline 11 & Nicosulfuron $0,040(\mathrm{POS})+\mathrm{CM}+[\mathrm{MSMA}+$ Diuron 1,44 + 1,0 (JD)] & $100,0 \mathrm{a}$ & $100,0 \mathrm{a}$ & $100,0 \mathrm{a}$ & $100,0 \mathrm{a}$ \\
\hline 12 & Testemunha (sem capina) & $0,0 \mathrm{~d}$ & $0,0 \mathrm{e}$ & $0,0 \mathrm{e}$ & $0,0 \mathrm{c}$ \\
\hline & Tratamentos (F) & $75,12^{*}$ & $16,44^{*}$ & $39,55^{*}$ & $737,92 *$ \\
\hline & C.V. $(\%)$ & 6,75 & 23,13 & 14,97 & 2,02 \\
\hline & d.m.s. $(5 \%)$ & 15,902 & 41,714 & 26,198 & 5,376 \\
\hline
\end{tabular}

* significância ao nivel de $1 \%$ de probabilidade

Médias nas colunas seguidas de mesma letra, não diferem significativamente entre si. 
TABELA 10. Resultados para produção de algodão em caroço após o plantio (D.A.P.) e a aplicação de herbicidas em PPI (pré-plantio incorporado), PRE (pré-emergência), POS (pós-emergência) e JD (jato dirigido) na cultura do algodão (Bariri - SP, 1994/95).

\begin{tabular}{lll}
\hline & \multicolumn{1}{c}{ Tratamentos } & Produção \\
\hline 1 & Trifluralina 1,068 (PPI) + Alachlor 1,584 (PRE) + CM + Diuron 1,5 (JD) & 1875,5 abc \\
2 & Trifluralina 1,068 (PPI) + Alachlor 1,584 (PRE) + CM + [Lactofen+Cyanazine 0,18 + 1,5 (JD)] & $1458,3 \mathrm{c}$ \\
3 & Trifluralina 1,068 (PPI) + Alachlor 1,584 (PRE) + CM + [Lactofen+Diuron 0,18 + 1,5 (JD)] & $1805,5 \mathrm{abc}$ \\
4 & Trifluralina 1,068 (PPI) + MSMA 1,68 (POS) + CM + Diuron 1,5 (JD) & $1666,6 \mathrm{bc}$ \\
5 & Trifluralina 1,068 (PPI) + Alachlor 1,584 (PRE) + CM + [MSMA+Cyanazine 1,44 + 1,0 (JD)] & $2150,3 \mathrm{abc}$ \\
6 & Trifluralina 1,068 (PPI) + Alachlor 1,584 (PRE) + MSMA 1,68 (POS) + CM + [MSMA+Diuron 1,44 + 1,0 (JD)] & $1805,5 \mathrm{abc}$ \\
7 & Trifluralina 1,068 (PPI) + Alachlor 1,584 (PRE) + CM + [MSMA+Lactofen 1,44 + 0,18 (JD)] & $2638,9 \mathrm{a}$ \\
8 & Trifluralina 1,068 (PPI) + Alachlor 1,584 (PRE) + MSMA 1,68 (POS) + CM + [MSMA+Diuron 1,68 + 1,0 (JD)] & $2152,8 \mathrm{abc}$ \\
9 & Nicosulfuron 0,020 (POS) + CM + [MSMA+Diuron 1,44 + 1,0 (JD)] & $442,3 \mathrm{~d}$ \\
10 & Nicosulfuron 0,030 (POS) + CM + [MSMA+Diuron 1,44 + 1,0 (JD)] & $501,0 \mathrm{~d}$ \\
11 & Nicosulfuron 0,040 (POS) + CM + [MSMA+Diuron 1,44 + 1,0 (JD)] & $496,3 \mathrm{~d}$ \\
12 & Testemunha (sem capina) & $427,6 \mathrm{~d}$ \\
\hline & Tratamentos (F) & $24,11^{*}$ \\
& C.V. (\%) & 19,47 \\
d.m.s. (5\%) & 703,4 \\
\hline
\end{tabular}

* significância ao nivel de $1 \%$ de probabilidade

Médias nas colunas seguidas de mesma letra, não diferem significativamente entre si. 
os tratamentos com M.S.M.A. em pós, e de quase forte a muito forte para os tratamentos com nicosulfuron aos 15 D.A.T.. Nas demais avaliações todos os tratamentos mostraram efeito nulo, exceto os sistemas com nicosulfuron que permaneceram com fitotoxicidade entre leve a moderada aos 89 D.A.T..

Os sistemas herbicidas compostos por aplicação de trifluralina em PPI, alachlor em pré, M.S.M.A. em pós (associado ou não ao préemergente), cultivo mecânico ao redor dos 50 a 60 D.A.P. e a aplicação de jato dirigido de diuron, lactofen + cyanazine, lactofen + diuron, M.S.M.A. + cyanazine, M.S.M.A. + lactofen e M.S.M.A. + diuron, propiciaram de forma geral controle das principais plantas daninhas, presentes na área experimental.

Os tratamentos com nicosulfuron, devido ao estádio no qual foi aplicado em pósemergência, além da alta fitotoxicidade apresentada, mostrou deficiência no nível de controle, principalmente para as duas menores doses e baixa produtividade em geral, devendo ser melhor estudada as épocas de sua aplicação para viabilizar seu uso nesta cultura. No entanto, Murai et al. (1995) relatam que o herbicida nicosulfuron é efetivo em diferentes plantas daninhas, como espécies anuais de gramíneas, folhas largas e espécies perenes de gramíneas a extremamente baixa dose de $40 \mathrm{~g}$ i.a./ha.

Os dados de produção mostraram melhor produtividade para o sistema de trifluralina (PPI), alachlor (PRE), CM, M.S.M.A. + lactofen com $2.638,9 \mathrm{~kg}$ de algodão em caroço/ha. Os outros dois tratamentos com produtividade superior a $2.000 \mathrm{~kg} / \mathrm{ha}$ foram trifluralina (PPI), alachlor (PRE), M.S.M.A. (POS), CM e M.S.M.A. + diuron e os mesmos tratamentos em PPI e pré, CM e M.S.M.A. + cyanazine.

Segundo Beltrão et al. (1983) o diuron é um dos herbicidas mais recomendados e utilizados no controle das plantas daninhas na cultura do algodão isolado, combinado ou misturado com outros herbicidas. Cruz \& Toledo (1982) verificaram que diuron a $1,0 \mathrm{~kg} / \mathrm{ha}+$ alachlor a
3,0 1/ha controlaram bem as plantas daninhas na cultura do algodão.

Victoria Filho et al. (1982) verificaram controles excelentes de monocotiledôneas e dicotiledôneas com a mistura de trifluralina a $1,00 \mathrm{~kg} / \mathrm{ha}$ e diuron a $1,2 \mathrm{~kg} / \mathrm{ha}$ em pré-plantio incorporado, em solo barrento. Thompson (1989) relata que o herbicida trifluralina é largamente utilizado no controle de plantas daninhas na cultura de algodão, sendo eficiente quando utilizado sozinho; mas, aumentando sua eficiência quando em mistura com diuron.

Cruz \& Leiderman (1978) verificaram controles excelentes de monocotiledôneas e dicotiledôneas com misturas de tanque de M.S.M.A. e diuron, aplicados em pré-emergência.

Estudos posteriores deverão determinar a possibilidade de substituir o cultivo mecânico, somente pela aplicação de herbicidas em jato dirigido, fato que possibilitaria facilidades de trabalho e redução de custos.

Os dados obtidos no presente experimento, permitem recomendar os herbicidas, cujos resultados foram positivos no sistema de herbicidas (PPI, PRE, POS, CM e JD) na cultura de algodão, tendo em vista sua eficiência demonstrada no controle das plantas daninhas e a segurança para a cultura.

\section{LITERATURA CITADA}

ALVES, A., FORSTER, R. Variações nos métodos de aplicação de herbicidas Diuron e Trifluralina na cultura do algodoeiro. In: SEMINÁRIO BRASILEIRO DE HERBICIDAS E ERVAS DANINHAS, 6, Sete Lagoas, 1968. Anais ... Sete Lagoas, S.B.H.E.D., 1968. 51p.

\section{ASOCIACION LATINOAMERICANA DE} MALEZAS. Recomendaciones sobre unificacion de los sistemas de evaluacion em ensayos de control de malezas. ALAM, v.1, n.1, p.35-38, 1974. 
BANZATTO, D.A., KRONKA, S.N. Experimentação agrícola. Jaboticabal, FUNEP, 1989. 247p.

BELTRÃO, N.E.M., SILVA, J.F., SILVEIRA, A.J., SEYDANA, C.S., COSTA, L.M., OLIVA, M.A. Comportamento do algodoeiro herbáceo (Gossypium hirsutum L. Hutch) e controle das plantas daninhas com o uso de herbicidas diuron $\mathrm{e}$ sethosydim. Planta Daninha, v.6, n.1, p.58-71, 1983.

CRUZ, L.S.P., LEIDERMAN, L. Aplicação de misturas de diuron com M.S.M.A. e com paraquat no controle de plantas daninhas de folhas largas em cultura de algodão (Gossypium hirsutum L.). Planta Daninha, v.1, n.1, p.45-50, 1978.

CRUZ, L.S.P., TOLEDO, N.M.P. Aplicação préemergente de misturas de alachlor com diuron e cyanazine para controle de plantas daninhas em algodão "IAC 17". Planta Daninha, v.5, n.2, p.57-61, 1982.

EUROPEAN WEED RESEARCH COUNCIL. Report of the $3^{\text {th }}$ and $4^{\text {th }}$ meetings of EWRC - Comittee of methods in weed research. Weed Res., v.4, n.1, p.88, 1964.

LACA-BUENDIA, J.P. Controle de plantas daninhas na cultura algodoeira. Inf. Agropec., v.92, n.8, p.28-33, 1992.

LEIDERMAN, L., SANTOS, C.A.L., SILVEIRA, R.I. Aplicação de herbicidas em algodão em três regiões do Estado de São Paulo. O Biológico, v.31, n.8, p.168-175, 1965.

LEPSCH, I.F., OLIVEIRA, J.B. Explicações sumárias sobre a nova nomenclatura das legendas de mapas pedológicos de São Paulo. Bol. Téc. Inst. Agron. Campinas, n.117, 1987. 36p.

MONTANDON, J.L. Delta agricultural digest. Clarksdale Mississipi, Ed. Montandon, 1993. 207p.

MURAI, S., HAGA, T., SAKASHITA, N., NAKAMURA, Y., HONDA, C., HONZAWA, S., KIMURA, F., TSUJII, Y., NISHIYAMA, R. Synthesis and herbicidal activity of sulfonylureas; SL-950 and its related compounds. J. Pesticides Sci., v.20, p.453-462, 1995.

OLIVEIRA, J.B., ALMEIDA, C.L.F., PRADO, H. Levantamento pedológico semidetalhado do Estado de São Paulo - Quadrícola de Jaú - SF.22.Z.B.11. Convênio EMBRAPA - Secretaria da Agricultura de São Paulo, 1982. 34p.

THOMPSON, W.T. Agricultural chemicals. USA, Thompson Publications, 1989. 330p.

VICTORIA FILHO, R., CRUZ, L.S.P., GARCIA, I. Efeitos de misturas de dinitranine e diuron em pré-plantio incorporado na cultura do algodão (Gossypium hirsutum L.). Planta Daninha, v.5, n.1, p.5-64, 1982. 